
\title{
25 Research Square \\ The influence of different glucose tolerance on QTC interval: a population-based study
}

\section{Ning Lin}

Shanghai Jiao Tong University School of Medicine

Hongmei Zhang

Shanghai Jiaotong University School of Medicine Xinhua Hospital

\section{Xiaoyong Li}

Shanghai Jiaotong University School of Medicine

\section{Yixin Niu}

Shanghai Jiaotong University School of Medicine Xinhua Hospital

\section{Hongxia Gu}

Shanghai Jiaotong University school of medicine Xinhua Hospital Chongming Branch

\section{Shuai Lu}

Shanghai Jiaotong University School of Medicine Xinhua Hospital Chongming Branch

\section{Zhen Yang}

Shanghai Jiaotong University School of Medicine Xinhua Hospital

\section{Li Qin}

Shanghai Jiaotong University School of Medicine

\section{Qing Su ( $\nabla$ suqing@xinhuamed.com.cn )}

Shanghai Jiao Tong University School of Medicine https://orcid.org/0000-0001-7945-1258

\section{Original investigation}

Keywords: QTc interval, abnormal glucose metabolism, type 2 diabetes

Posted Date: October 19th, 2021

DOI: https://doi.org/10.21203/rs.3.rs-961619/v1

License: (c) (i) This work is licensed under a Creative Commons Attribution 4.0 International License. Read Full License 


\section{Abstract \\ Background}

Corrected QT (QTc) interval has been reported to be associated with type 2 diabetes. This study aimed to explore the relationship between different glucose tolerance and QTc interval among middle-aged and older Chinese individuals.

\section{Methods}

We conducted a cross-sectional analysis that included 9898 subjects (3194 men and 6704 women) in a Chinese population. Glucose tolerance was studied during OGTT. Insulin, blood pressure, HbA1c, serum lipids, hepatic transaminases and waist-to-hip ratio were assessed.The QTC interval was derived from ECG recordings, and the subjects were stratified based on different glucose tolerance.

\section{Results}

QTc interval levels were increased significantly in the subjects with abnormal glucose metabolism compared with the normal glucose regulation group. Multiple regression analyses QTc interval was significantly associated with fasting plasma glucose, 2-h OGTT plasma glucose and HbA1c. The odds ratios of prolonged QTc was 1.396 for IFG/IGT (95\% Cl: 0.126-1.730), 1.342 for type 2 diabetes (95\% Cl: $0.142-1.577)$ after all the potential confounders were adjusted.

\section{Conclusions}

IGR and diabetes are associated with prolonged QTc interval among middle-aged and older Chinese individuals. Abnormal glucose regulation can be used to monitor QTc interval in the population.

\section{Background}

The risk of sudden death is increased in patients with diabetes mellitus and impaired glucose tolerance[1, 2]. Abnormal cardiac autonomic nervous system function is one of the most significant complications of diabetes[3].The QT interval is defined as the total time required for ventricular myocardial depolarization and repolarization[4]. Corrected QT (QTC) is very important for the prediction, risk assessment and treatment of ventricular arrhythmias[2].The relation between a prolonged QTc interval and an increased risk of sudden death has been traditionally explored in familial long QT syndrome sudden cot death, and congestive heart failure, as well as in adults with diabetes mellitus[2,5-8]ष

Although several researches describe the relationship between QTc interval prolongation, diabetic complications, and an increased mortality rate in adults[5, 9-11], the influence of abnormal glucose metabolism on QTc interval has not been fully clarified. Previous studies have confirmed that the prevalence of prolonged QTc interval is increased in people with type 1 and type 2 diabetes as compared to non-diabetics, and the abnormalities in cardiac repolarisation may be caused by complications of diabetes rather than diabetes in itself $[5,10,12,13]$. Most 
recently, Kurnaz E et al. found that QTc prolongation is already existent in a significant proportion of children and adolescents with newly diagnosed type 1diabetes[14].

However, up to recently, only few studies have reported conflicting data on the relationship between QTc interval and glucose metabolism regulation, especially the status of impaired glucose regulation (IGR) $[5,6,10,12]$. In this study, we aimed to investigate the influence of different glucose tolerance on QTc interval in a cross-sectional study of Chinese individuals aged $40-70$ years.

\section{Methods}

Study population

The present study is one part of the risk evaluation of cancers in Chinese diabetic individuals, i.e., the longitudinal study (REACTION) which was a population-based cross-sectional study among middle-aged and elderly Chinese individuals in 25 communities across mainland China. The studied individuals aged 40 to 70 years old. The details of the study design have been described beforehand $[15,16]$. All participants came from the Chongming District in Shanghai, China. A total of 9930 suitable subjects were recruited. After the exclusion of 1940 individuals with selfreported coronary heart disease, or taking medicine affecting heart rhythm, 7990 subjects (2489 men and 5501 women) were found to be suitable for the current analysis. Written informed consent was gained from all of the participants. Approval was given by the Institutional Review Board of Xinhua Hospital affiliated with Shanghai Jiaotong University School of Medicine.

Data collection

A standardized questionnaire was used by trained physicians to collect the baseline data. The measurements of weight, height, waist circumference, hip circumference and blood pressure have been described previously. Smoking (yes/no) and alcohol drinking (yes/no) were estimated with an interview preceding the physical examination. Body mass index (BMI) was calculated as weight in kilograms divided by the square of height in meters.

A 12-lead ECG was recorded at a paper speed of $50 \mathrm{~mm} / \mathrm{s}$ on a six-channel recorder. The QT interval was measured from the beginning of the QRS complex to the end of the T-wave. The QT interval corrected for the previous cardiac R-R cycle length (QTC) was calculated according to the formula proposed previously and known as Bazett's formula: QTc = QT/(RR) 1/2[17]. QTc is the mean of QTc from five consecutive cycles in lead V5. QTc $₫ 440$ ms was considered abnormally prolonged[18].

After overnight fasting for at least 10 hours, fasting and $2 \mathrm{~h}$ OGTT (oral glucose tolerance test) blood samples were collected from all the participants in tubes containing EDTA and were centrifuged at $4^{\circ} \mathrm{C}$ and stored at $-80^{\circ} \mathrm{C}$ till analysis. The fasting glucose, $2 \mathrm{~h}$ OGTT plasma glucose, triglycerides, total cholesterol (TC), high-density lipoprotein (HDL) cholesterol, low-density lipoprotein (LDL) cholesterol, and serum creatinine (Scr) were measured on an automatic analyzer (Hitachi 7080; Tokyo, Japan). The homeostasis model assessment of insulin resistance (HOMA-IR) was calculated in the light of the equation described by Matthews et al[19].

Definitions of impaired glucose regulation and type 2 diabetes

Impaired glucose regulation was defined as impaired fasting glucose (IFG, fasting plasma glucose level $\geq 6.1$ and $<$ $7.0 \mathrm{mmol} / \mathrm{I}$ ) and/or impaired glucose tolerance (IGT, 2h OGTT plasma glucose level $\geq 7.8$ and $<11.1 \mathrm{mmol} / \mathrm{I}$ ). Isolated IFG: fasting plasma glucose $\geq 6.1 \mathrm{mmol} / \mathrm{I}$ and $<7.0 \mathrm{mmol} / \mathrm{I}$ and a $2 \mathrm{~h}$ OGTT plasma glucose $<7.8 \mathrm{mmol} / \mathrm{l}$. 
Isolated IGT: $2 \mathrm{~h}$ OGTT plasma glucose $\geq 7.8 \mathrm{mmol} / \mathrm{I}$ and $<11.1 \mathrm{mmol} / \mathrm{l}$ and a fasting glucose $<6.1 \mathrm{mmol} / \mathrm{l}$. IFG/IGT: fasting plasma glucose between $6.1 \mathrm{mmol} / \mathrm{l}$ and $6.9 \mathrm{mmol} / \mathrm{l}$ and a $2 \mathrm{~h}$ OGTT plasma glucose $7.8 \mathrm{mmol} / \mathrm{I}$ to $11.0 \mathrm{mmol} / \mathrm{l}$. Type 2 diabetes was diagnosed by the 1999 World Health Organization criteria (fasting plasma glucose level $\geq 7.0 \mathrm{mmol} / \mathrm{l}$ and/or a $2 \mathrm{~h}$ OGTT plasma glucose level $\geq 11.1 \mathrm{mmol} / \mathrm{l}[20]$. A fasting glucose level lower than $6.1 \mathrm{mmol} / \mathrm{I}$ and a $2 \mathrm{~h}$ OGTT plasma glucose level below $7.8 \mathrm{mmol} / \mathrm{I}$ were defined as normal glucose regulation (NGR).

\section{Statistical analysis}

Data management and statistical analysis were performed with the SPSS Statistical Package (version 22.0; SPSS Inc., Chicago, IL). Normally distributed data were expressed as means \pm SD, whereas variables with a skewed distribution were reported as median (inter-quartile range). Comparisons of means and proportions were performed

with the t-test and $\chi^{2}$ tests, respectively. Crude and partial correlation and multivariable stepwise regression analysis were used to investigate the association of QTc interval with cardiovascular and metabolic related parameters. A multiple linear regression analysis was performed to determine the associations of different glucose tolerance with QTc interval. Finally, a multivariate logistic regression model was used to evaluate the ORs and $95 \%$ Cls of different glucose tolerance for prolonged QTc. The potential confounders including age, sex, insulin, SBP, DBP, CHOL, TG, smoking, and drinking were adjusted. $\mathrm{P}<0.05$ was considered statistically significant.

\section{Results}

\section{Clinical characteristics of participants}

The clinical characteristics of the participants stratified by glucose tolerance are summarized in Table 1. The study involved 3412 participants with NGR, 2475 with IGR and 1833 with type 2 diabetes. Among the participants with IGR, 773 (9.67 \%) had isolated IFG, 1255 (15.7\%) had isolated IGT, and 717 (8.97\%) had combined IFG/IGT. The NGR and diabetes groups had, respectively, the most favorable and unfavorable metabolic profiles. Across different glucose tolerance, the subjects with higher blood glucose levels were more likely to be older, have higher waist to hip ratio, higher lipid profiles, and higher levels of blood pressure. Some liver and kidney function indicators (such as AST, ALT, GGT, SCr) between groups were different significantly. In addition, the levels of QTc interval were significantly increased in the subjects with isolated IFG, isolated IGT, combined IFG and IGT, and type 2 diabetes compared with the subjects with normal glucose regulation $(416.63 \pm 24.79,418.53 \pm 24.20,419.2 \pm 27.35$, and $420.79 \pm 25.49$, respectively, vs. $415.6 \pm 24.30, p<0.05$ ) (Fig. 1). QTc interval was positively associated with abnormal glucose metabolism. 
Table 1

Clinical and laboratory characteristics according to different glucose tolerance.

\begin{tabular}{|c|c|c|c|c|c|c|}
\hline Characteristics & DM & IFG+IGT & IGT & IFG & NGT & $\begin{array}{l}P \\
\text { value }\end{array}$ \\
\hline $\mathrm{N}$ & 1833 & 717 & 1255 & 773 & 3412 & \\
\hline Age (yr) & $58.21 \pm 7.20$ & $56.86 \pm 7.46$ & $55.22 \pm 7.60$ & $55.56 \pm 7.60$ & $53.47 \pm 7.79$ & $<0.001$ \\
\hline Gender (M/F) & 747/1086 & $253 / 464$ & $307 / 948$ & $316 / 457$ & $866 / 2546$ & $<0.001$ \\
\hline QTC & $420.79 \pm 25.49$ & $419.20 \pm 27.35$ & $418.53 \pm 24.20$ & $416.63 \pm 24.79$ & $415.60 \pm 24.30$ & $<0.001$ \\
\hline BMI (kg/m2) & $25.18 \pm 3.38$ & $25.06 \pm 3.53$ & $24.91 \pm 10.43$ & $24.38 \pm 3.35$ & $23.74 \pm 3.25$ & $<0.001$ \\
\hline WHR & $0.90 \pm 0.07$ & $0.89 \pm 0.09$ & $0.88 \pm 0.10$ & $0.88 \pm 0.08$ & $0.86 \pm 0.08$ & $<0.001$ \\
\hline $\mathrm{SBP}(\mathrm{mm} \mathrm{Hg})$ & $136.51 \pm 19.00$ & $133.38 \pm 17.22$ & $127.74 \pm 17.37$ & $130.81 \pm 18.01$ & $123.80 \pm 18.42$ & $<0.001$ \\
\hline DBP $(\mathrm{mm} \mathrm{Hg})$ & $81.78 \pm 10.37$ & $82.10 \pm 9.51$ & $79.97 \pm 9.89$ & $80.87 \pm 9.66$ & $77.79 \pm 10.52$ & $<0.001$ \\
\hline FBG (mmol/l) & $8.05 \pm 2.59$ & $6.38 \pm 0.24$ & $5.57 \pm 0.34$ & $6.34 \pm 0.22$ & $5.41 \pm 0.37$ & $<0.001$ \\
\hline PBS (mmol/l) & $13.41 \pm 4.83$ & $9.09 \pm 0.89$ & $8.92 \pm 0.84$ & $6.28 \pm 1.02$ & $6.08 \pm 1.05$ & $<0.001$ \\
\hline HbA1C (\%) & $6.91 \pm 1.61$ & $5.83 \pm 0.41$ & $5.74 \pm 0.39$ & $5.71 \pm 0.41$ & $5.60 \pm 0.40$ & $<0.001$ \\
\hline Insulin(pmol/l) & $8.92 \pm 3.24$ & $8.10 \pm 3.89$ & $7.27 \pm 3.66$ & $7.26 \pm 3.35$ & $6.27 \pm 3.07$ & $<0.001$ \\
\hline HOMA-IR & $3.23 \pm 0.94$ & $2.36 \pm 0.92$ & $1.85 \pm 0.93$ & $2.11 \pm 0.97$ & $1.56 \pm 0.78$ & $<0.001$ \\
\hline $\mathrm{HDL}(\mathrm{mmol} / \mathrm{L})$ & $1.22 \pm 0.32$ & $1.23 \pm 0.33$ & $1.22 \pm 0.28$ & $1.28 \pm 0.33$ & $1.25 \pm 0.32$ & $<0.001$ \\
\hline LDL (mmol/L) & $2.70 \pm 0.79$ & $2.71 \pm 0.73$ & $2.60 \pm 0.73$ & $2.72 \pm 0.76$ & $2.51 \pm 0.74$ & $<0.001$ \\
\hline $\mathrm{CHO}(\mathrm{mmol} / \mathrm{L})$ & $4.86 \pm 1.08$ & $4.85 \pm 0.99$ & $4.61 \pm 0.99$ & $4.84 \pm 1.02$ & $4.45 \pm 0.98$ & $<0.001$ \\
\hline TG (mmol/L) & $2.08 \pm 1.71$ & $2.02 \pm 1.11$ & $1.73 \pm 0.89$ & $1.70 \pm 0.91$ & $1.38 \pm 0.71$ & $<0.001$ \\
\hline $\operatorname{ALT}(\mathrm{U} / \mathrm{L})$ & $20.86 \pm 10.07$ & $19.04 \pm 10.31$ & $16.99 \pm 8.80$ & $17.28 \pm 8.04$ & $13.97 \pm 7.55$ & $<0.001$ \\
\hline AST (U/L) & $22.80 \pm 10.95$ & $21.88 \pm 10.16$ & $20.57 \pm 9.22$ & $21.36 \pm 8.76$ & $18.89 \pm 9.03$ & $<0.001$ \\
\hline GGT (U/L) & $38.96 \pm 14.55$ & $33.49 \pm 32.77$ & $28.55 \pm 36.90$ & $31.04 \pm 14.14$ & $22.05 \pm 11.11$ & $<0.001$ \\
\hline Scr (umol/L) & $68.71 \pm 17.48$ & $68.07 \pm 19.95$ & $64.03 \pm 12.94$ & $67.91 \pm 14.05$ & $63.34 \pm 12.06$ & $<0.001$ \\
\hline $\begin{array}{l}\text { Smoking } \\
\text { (n/total) }\end{array}$ & $492 / 3412$ & $161 / 773$ & $156 / 1255$ & $115 / 717$ & $359 / 1833$ & $<0.001$ \\
\hline $\begin{array}{l}\text { Drinking } \\
\text { (n/total) }\end{array}$ & $705 / 3412$ & $222 / 773$ & $227 / 1255$ & $179 / 717$ & 449/1833 & $<0.001$ \\
\hline \multicolumn{7}{|c|}{ Values are mean \pm SD or median (interquartile range) or number (proportion) } \\
\hline $\mathrm{p}$ values were & e ANOVA or & melyon & e five groups & & & \\
\hline
\end{tabular}

Association between QTc interval and clinical characteristics

Table 2 presents the results of correlation analyses of QTc interval with metabolic related parameters. QTc levels correlated positively with age, gender, WHR, SBP, DBP, PBS, FBS, HBA1c, insulin, HOMA-IR, LDL, CHO, TG, smoking, 
drinking. After adjusting for age and gender, WHR, SBP, DBP, PBS, FBS, HA1c, insulin, HOMA-IR, CHO, TG was still positively associated with QTc interval.

Table 2

Crude and partial correlation between QTc and clinical parameters in the studied subjects

\begin{tabular}{|c|c|c|}
\hline Variable & Crude r & Partial $r^{\dagger}$ \\
\hline Age (year) & $0.139 * *$ & - \\
\hline Gender & $0.148 * \star$ & - \\
\hline BMI (kg/m2) & 0.01 & 0.006 \\
\hline WHR & $0.031^{*}$ & $0.039 *$ \\
\hline $\mathrm{SBP}(\mathrm{mmHg})$ & $0.094^{\star \star}$ & $0.085^{\star \star}$ \\
\hline $\mathrm{DBP}(\mathrm{mmHg})$ & $0.054^{\star *}$ & 0.081 ** \\
\hline PBS (mmol/L) & $0.091 * *$ & $0.075^{\star \star}$ \\
\hline FBS (mmol/L) & $0.084 * *$ & $0.083^{\star *}$ \\
\hline HbA1c (\%) & $0.074 * \star$ & $0.053^{\star \star}$ \\
\hline Insulin (pmol/L) & $0.061 * *$ & $0.045^{\star \star}$ \\
\hline HOMA_IR & $0.068^{\star *}$ & 0.054 ** \\
\hline $\mathrm{HDL}(\mathrm{mmol} / \mathrm{L})$ & 0.014 & 0.004 \\
\hline LDL (mmol/L) & $0.046^{\star *}$ & 0.021 \\
\hline $\mathrm{CHO}(\mathrm{mmol} / \mathrm{L})$ & $0.068 * *$ & $0.041^{\star *}$ \\
\hline TG (mmol/L) & $0.062 * \star$ & 0.071 ** \\
\hline Smoking & $-0.106^{* *}$ & -0.002 \\
\hline Drinking & $-0.083^{\star \star}$ & -0.007 \\
\hline \multicolumn{3}{|c|}{${ }^{*} \mathrm{p}<0.01,{ }^{* *} \mathrm{p}<0.001$, tadjusted for age, gende } \\
\hline
\end{tabular}

Determinants of QTc levels

Table3 demonstrated variables independently associated with QTc interval. Age, gender, SBP, DBP, PBS, FBS, HBA1c, TG were independent determinants for QTc interval. 
Table 3

Multiple stepwise regression analysis showing variables independently associated with QTC.

\begin{tabular}{|llll|}
\hline Independent variables & Standardized $\boldsymbol{\beta}$ & $\mathbf{t}$ & $\mathbf{P}$ value \\
\hline Age & 0.125 & 10.615 & $<0.001$ \\
\hline Sex & 0.192 & 17.004 & $<0.001$ \\
\hline FBS & 0.065 & 5.706 & $<0.001$ \\
\hline PBS & 0.052 & 4.557 & $<0.001$ \\
\hline HbA1c & 0.043 & 3.819 & $<0.001$ \\
\hline SBP & 0.044 & 3.007 & 0.003 \\
\hline DBP & 0.036 & 2.498 & 0.012 \\
\hline TG & 0.049 & 4.358 & $<0.001$ \\
\hline $\begin{array}{l}\text { The analysis also included CHO, WHR, Insulin, BMl, smoking, and drinking which were all excluded from the } \\
\text { model. }\end{array}$ & & \\
\hline
\end{tabular}

Associations between different glucose tolerance and QTc interval

To further explore the relationship between different glucose tolerance and QTc interval, multiple regression analyses were performed. The analyses revealed that solated IGT, combined IFG/IGT and type 2 diabetes are significantly associated with QTc interval after adjusting for all potentially confounders (age, sex, insulin, SBP, DBP, CHOL, TG, smoking, and drinking) (Table4).

Table 4

Adjusted associations with QTc according to different glucose tolerance.

\begin{tabular}{|lllll|}
\hline \multicolumn{1}{|c}{ Glucose tolerance category } & Standardized $\boldsymbol{\beta}$ & $\mathbf{t}$ & P value \\
\hline Model 1 & NGT & reference & 1.042 & 0.298 \\
& IFG & 0.012 & 3.561 & $<0.001$ \\
& IGT & 0.043 & 3.522 & $<0.001$ \\
& IFG+IGT & 0.041 & 7.196 & $<0.001$ \\
& DM & 0.087 & & \\
\hline Model 2 & NGT & reference & 1.938 & 0.053 \\
& IFG & 0.027 & 3.043 & 0.002 \\
& IGT & 0.044 & 2.279 & 0.023 \\
& IFG+IGT & 0.032 & 5.049 & $<0.001$ \\
& DM & & \\
\hline Model 1 not adjusted. & 0.076 & & \\
\hline Model 2 adjusted for age, sex, insulin, SBP, DBP, CHOL, TG, smoking, and drinking. \\
\hline
\end{tabular}


In the present study, we consider QTc $₫ 440$ ms as abnormally prolonged. Taken NGR as reference, isolated IGT, combined IFG and IGT, and type 2 diabetes were all risk factors for prolonged QTC. OR of IGT for QTc prolongation was 1.131 (95\% Cl 1.059-1.356), OR of IFG+IGT for QTc prolongation was 1.396 (95\% Cl 1.126-1.73), and OR of T2DM for QTc prolongation was 1.34 (95\% Cl 1.142-1.577), respectively ( $P$ for trend < 0.001$)$ after adjusting for age, sex, insulin, SBP, DBP, CHOL, TG, smoking, and drinking (Table5).

Table 5

Adjusted ORs and 95\% Cls for prolonged QTc according to different glucose tolerance.

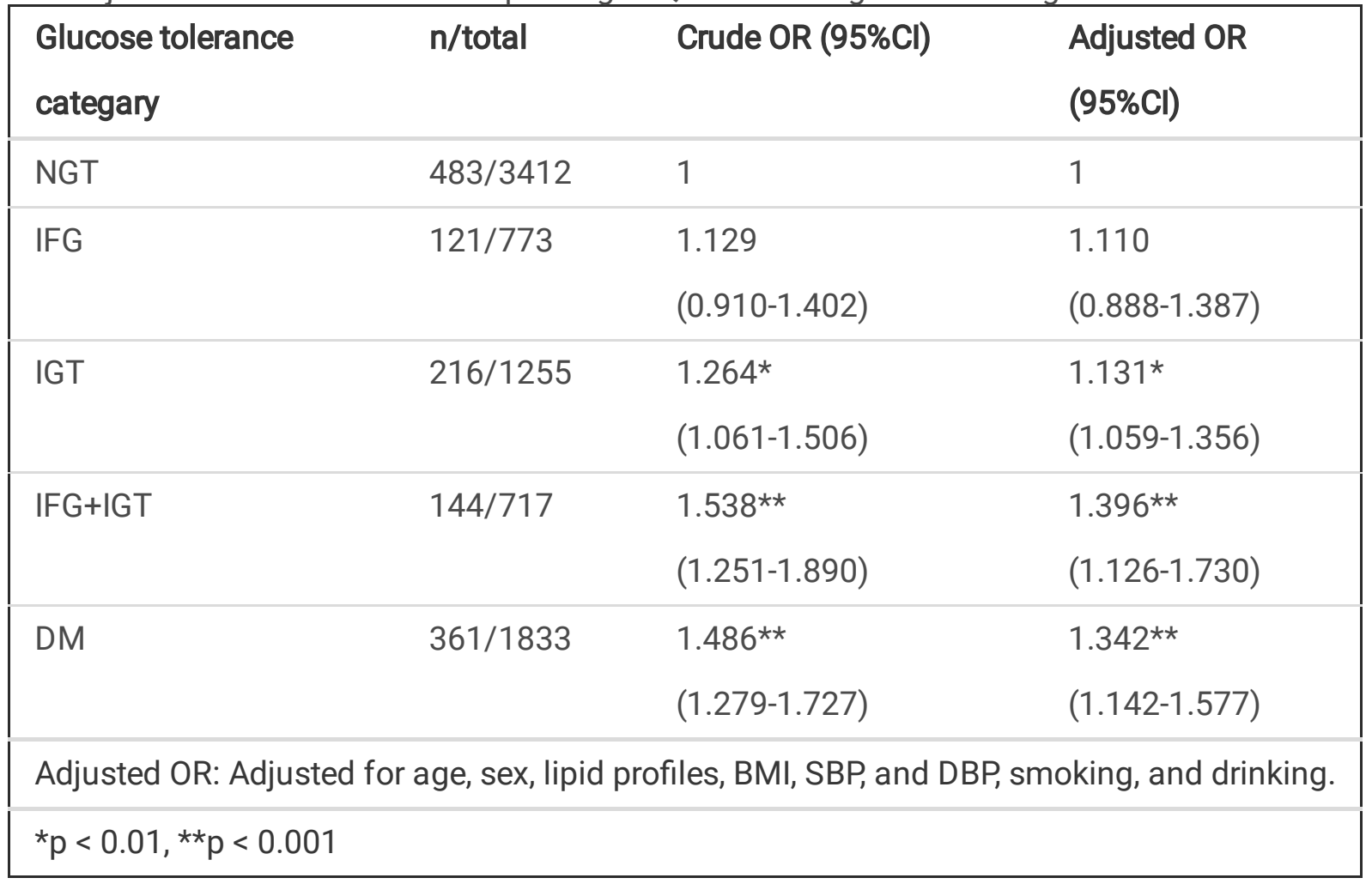

\section{Discussion}

The main finding of this study is the effect of different glucose tolerance on QTc interval. QTc interval prolongation is more common in patients with isolated IGT, combined IFG and IGT, and type 2 diabetes compared to participants with NGR. Moreover, this association is independent of potential confounding covariates. Given the evidence that a prolonged QTc interval is related to adverse cardiovascular event, our results indicate that persons with IGR and diabetes may be at a high risk of arrhythmia, sudden death, and cardiovascular disease (CVD) mortality.

The QTc prolongation is reported to be an independent risk factor for coronary heart disease in type 1 and type 2 diabetes and has been described to be a prominent predictor of cardiac death even in newly diagnosed type 2 diabetes[21]. The presence of cardiovascular autonomic neuropathy (CAN) is considered to be a strong indicator of mortality. Additionally, lots of studies have demonstrated that the QTc prolongation has direct detrimental effects on the deadly arrhythmias and risk of sudden death with myocardial ischemia $[6,13]$. The above studies support the role of QTc interval in predicting the risks of mortality, in this study, we demonstrated that QTc interval was prolonged in pre-diabetes and diabetes in middle-aged and elderly Chinese individuals. 
Previous published articles have reported the risk factors of prolonged QTc interval among patients with diabetes included age[13], gender[5], BMI[22], hypertension[23], insulin concentration and hyperglycemia[24]. In consistent with above studies, our analysis showed age, gender, SBP, DBP, PBS, FBS, HBA1c, TG were positively correlated with QTc interval. However, after further adjusting the related confounding variables, BMI, Insulin, HOMA-IR, HDL, LDL, $\mathrm{CHO}$ were no longer independently related to QTC interval. In agreement with our results, the EURODIAB Prospective Complications Study demonstrated that female sex, higher values of HbA1c and systolic blood pressure were associated with the higher incidence of prolonged QTc, whereas BMI and physical activity within the range of 21.5$23.2 \mathrm{~kg} / \mathrm{m}^{2}$ were displayed as protective factors[25].

Persons with diabetes mellitus have increased QTc interval[26]. We evaluated the association between different glucose tolerance and prolonged QTc interval using the logistic regression model. Our results agreed with the previous findings that patients with IGR, a pre-diabetic condition, were correlated with QTc interval[25, 27]. However, surprisingly, impaired fasting glucose was not found to influence QTc interval significantly, which is not in consistent with other studies[28,29]. The reason for this is probably because the participants in our study were from only one area and more women were recruited than man.

It was previously revealed that hyperglycemia may lead to QTc prolongation by several mechanisms including the stimulation of protein kinase $\mathrm{C}$ which causes reduced synthesis and release of endothelial derived nitric oxide[30]. This consequently results in a decrease in the activity of Na-K-ATPase. Perhaps the reduced nitric oxide bioavailability during hyperglycemia is responsible for decreased activity of $\mathrm{Ca}^{2 \rrbracket} \mathrm{ATPase}$ as well[31], an enzyme maintains a low concentration of $\mathrm{Ca}^{2 \mathbb{}}$ ions in the cell. Decreased activity of Na-K-ATPase increases the intracellular calcium concentration and extend QTc interval. There is also accumulating evidence suggests that a direct relationship between type 2 ryanodine receptor (RyR2) dysfunction in the endo/sarcoplasmic reticulum leading to altered intracellular calcium homeostasis, glucose intolerance, and impaired insulin secretion in patients with catecholaminergic polymorphic ventricular tachycardia[32]. Moreover, hyperglycemia is related to increased sympathetic activity as evident by increased plasma catecholamine concentrations[33]. Sympathetic stimulation unopposed by vagal activity may also induce ventricular electrical instability.

Impaired glucose regulation is widely considered with a relatively high risk for the future development of diabetes[34]. IGR should not be seen as an independent clinical entity but rather as a risk factor for diabetes and cardiovascular disease. Moreover, it has been well recognized that IGR is associated with hypertension, high triglycerides, low HDL cholesterol and visceral obesity. Therefore, elucidating the relationship of impaired glucose regulation and QTc interval and seeking effective management for all modifiable risk factors are of great importance.

In our study, pre-diabetes and diabetes appeare to be independent risk factors for QTc interval prolongation after controlling the potential cofounders. QTc interval is notably associated with glucose metabolism indices (including FPS, PPS, HbA1c). In view of this, it is reasonable to consider whether QTc interval alone should become a novel target for clinical intervention.

The advance of the present study is that it evaluated the relationship between pre-diabetes and diabetes and QTC interval in such a large-scale population. The results of our study support the concept that postprandial glucose level elevations may have an important role in the pathogenesis of cardiovascular complications[25]. The vital role of hyperglycemia, especially postprandial, as an essential risk factor for QTc prolongation has been approved in 
this study. In consistent with our results, postprandial glucose is considered to be a better predictive regarding cardiovascular mortality in comparison to fasting plasma glucose in the DECODE study [35].

Our study has some limitations. First, given the cross-sectional nature of the study design, a cause-effect relationship between abnormal glucose metabolism and QTc interval could not be verified. Further prospective studies are needed to analyze the potential pathophysiological mechanism of this correlation. Secondly, besides QTc interval, other markers of cardiac repolarization abnormalities, such as QT dispersion, were not evaluated. Thirdly, because our data were obtained from middle-aged and older subjects, and the gender proportions were unbalanced, it has yet to be seen whether our results can be generalized to younger populations or other ethnic groups. Finally, the QTc intervals were from one ECG which might not represent the true resting heart rate.

\section{Conclusions}

In summary, our findings suggested that increased risk of having abnormal glucose metabolism is intensively and independently associated with prolonged QTc interval in Chinese adults. As a conversant and accessible clinical parameter, abnormal glucose regulation can be used to monitor QTc interval in the population. Although longitudinal studies are needed, our results probably have vital public health meanings. Further research is still needed to verify whether glucose control among people with glucose intolerance may be useful in reducing QTc interval prolongation and ultimately reducing the risk of ventricular arrhythmia and sudden death.

\section{List Of Abbreviations}

QTc Corrected QT; IGR impaired glucose regulation; IFG impaired fasting glucose; IGT impaired glucose; tolerance; DM diabetes mellitus; WHR waist-to-hip ratio; BMI body mass index; SBP systolic blood pressure; DBP diastolic blood pressure; FPG fasting plasma glucose; 2hPPG 2-h post-load plasma glucose; HbA1c hemoglobin A1c; TC total cholesterol; TG triglycerides; LDL low-density lipoprotein-cholesterol; HDL high-density lipoprotein-cholesterol; IGR impaired glucose regulation; ALT alanine aminotransferase; AST aspertate aminotransferase; SCr serum creatinine.

\section{Declarations}

\section{Ethics approval and consent to participate}

Ethics Committee of Xinhua Hospital Affiliated to Shanghai Jiaotong University School of Medicine. The methods were carried out in accordance with the approved guidelines. Written informed consent was obtained from all participants before data collection.

\section{Consent for publication}

Not applicable.

\section{Availability of data and materials}

The datasets used and/or analyzed during the current study are available from the corresponding author on reasonable request. 
No potential conflicts of interest relevant to this article were reported.

\section{Funding}

This work was supported by the National Natural Science Foundation of China (81970669). Hospital Funded Clinical Research, Xin Hua Hospital Affiliated to Shanghai Jiao Tong University School of Medicine(19XHCR07B).

\section{Authors' contributions}

Lin N, and Zhang HM drafted the manuscript and performed the experiments. Su Q and Qin L conceived of and designed the study. Gu HX, Lu S and Qin L recruited the subjects, processed the samples, and contributed to the acquisition of the data. Li XY, and Niu YX analyzed the data. Yang Z revised the manuscript. All authors read and approved the final manuscript.

\section{Acknowledgments}

The authors gratefully acknowledge the support of the study participants, study staff, and partner organizations participating in the baseline survey and follow-up investigation.

\section{References}

1. Curb JD, Rodriguez BL, Burchfiel CM, Abbott RD, Chiu D, Yano K. Sudden death, impaired glucose tolerance, and diabetes in Japanese American men. Circulation. 1995;91(10):2591-5.

2. Svane J, Pedersen-Bjergaard U, Tfelt-Hansen J. Diabetes and the Risk of Sudden Cardiac Death. Curr Cardiol Rep. 2020;22(10):112.

3. Agashe S, Petak S. Cardiac Autonomic Neuropathy in Diabetes Mellitus. Methodist Debakey Cardiovasc J. 2018;14(4):251-6.

4. Rautaharju PM, Manolio TA, Psaty BM, Borhani NO, Furberg CD. Correlates of QT prolongation in older adults (the Cardiovascular Health Study). Cardiovascular Health Study Collaborative Research Group. The American journal of cardiology. 1994;73(13):999-1002.

5. Subbalakshmi NK, Adhikari PM, Sathyanarayana Rao KN, Jeganathan PS. Influencing factors of QTc among the clinical characteristics in type 2 diabetes mellitus. Diabetes Res Clin Pract. 2010;88(3):265-72.

6. Cox AJ, Azeem A, Yeboah J, Soliman EZ, Aggarwal SR, Bertoni AG, Carr JJ, Freedman BI, Herrington DM, Bowden DW. Heart rate-corrected QT interval is an independent predictor of all-cause and cardiovascular mortality in individuals with type 2 diabetes: the Diabetes Heart Study. Diabetes care. 2014;37(5):1454-61.

7. Van Niekerk C, Van Deventer BS, du Toit-Prinsloo L. Long QT syndrome and sudden unexpected infant death. J Clin Pathol. 2017;70(9):808-13.

8. Schwartz PJ, Wolf S. QT interval prolongation as predictor of sudden death in patients with myocardial infarction. Circulation. 1978;57(6):1074-7.

9. Yang XH, Su JB, Zhang XL, Zhao LH, Xu F, Wang XQ, Cheng XB. The relationship between insulin sensitivity and heart rate-corrected QT interval in patients with type 2 diabetes. Diabetol Metab Syndr. 2017;9:69.

10. Arildsen H, May O, Christiansen EH, Damsgaard EM. Increased QT dispersion in patients with insulin-dependent diabetes mellitus. Int J Cardiol. 1999;71(3):235-42. 
11. Veglio M, Sivieri R, Chinaglia A, Scaglione L, Cavallo-Perin P. QT interval prolongation and mortality in type 1 diabetic patients: a 5-year cohort prospective study. Neuropathy Study Group of the Italian Society of the Study of Diabetes, Piemonte Affiliate. Diabetes Care. 2000;23(9):1381-3.

12. Su JB, Yang XH, Zhang XL, Cai HL, Huang HY, Zhao LH, Xu F, Chen T, Cheng XB, Wang XQ, et al. The association of long-term glycaemic variability versus sustained chronic hyperglycaemia with heart ratecorrected QT interval in patients with type 2 diabetes. PloS one. 2017;12(8):e0183055.

13. Veglio M, Giunti S, Stevens LK, Fuller JH, Perin PC, Group EICS. Prevalence of Q-T interval dispersion in type 1 diabetes and its relation with cardiac ischemia: the EURODIAB IDDM Complications Study Group. Diabetes Care. 2002;25(4):702-7.

14. Kurnaz E, Erdeve SS, Ozgur S, Keskin M, Ozbudak P, Cetinkaya S, Aycan Z. Congenital long-QT syndrome in type 1 diabetes: a unique association. Turk J Pediatr. 2019;61(5):791-3.

15. Ning G, Reaction Study G. Risk Evaluation of cAncers in Chinese diabeTic Individuals: a IONgitudinal (REACTION) study. Journal of diabetes. 2012;4(2):172-3.

16. Qin L, Yang Z, Gu H, Lu S, Shi Q, Xing Y, Li X, Li R, Ning G, Su Q. Association between serum uric acid levels and cardiovascular disease in middle-aged and elderly Chinese individuals. BMC Cardiovasc Disord. 2014;14:26.

17. Luo S, Michler K, Johnston P, Macfarlane PW. A comparison of commonly used QT correction formulae: the effect of heart rate on the QTC of normal ECGs. J Electrocardiol. 2004;37 Suppl:81-90.

18. Fagher K, Londahl M. The impact of metabolic control and QTc prolongation on all-cause mortality in patients with type 2 diabetes and foot ulcers. Diabetologia. 2013;56(5):1140-7.

19. Matthews DR, Hosker JP, Rudenski AS, Naylor BA, Treacher DF, Turner RC. Homeostasis model assessment: insulin resistance and beta-cell function from fasting plasma glucose and insulin concentrations in man. Diabetologia. 1985;28(7):412-9.

20. Gabir MM, Hanson RL, Dabelea D, Imperatore G, Roumain J, Bennett PH, Knowler WC. The 1997 American Diabetes Association and 1999 World Health Organization criteria for hyperglycemia in the diagnosis and prediction of diabetes. Diabetes Care. 2000;23(8):1108-12.

21. Rana BS, Lim PO, Naas AA, Ogston SA, Newton RW, Jung RT, Morris AD, Struthers AD. QT interval abnormalities are often present at diagnosis in diabetes and are better predictors of cardiac death than ankle brachial pressure index and autonomic function tests. Heart. 2005;91(1):44-50.

22. Festa A, D'Agostino R Jr, Rautaharju P, Mykkanen L, Haffner SM. Relation of systemic blood pressure, left ventricular mass, insulin sensitivity, and coronary artery disease to QT interval duration in nondiabetic and type 2 diabetic subjects. The American journal of cardiology. 2000;86(10):1117-22.

23. Sohaib SM, Papacosta O, Morris RW, Macfarlane PW, Whincup PH. Length of the QT interval: determinants and prognostic implications in a population-based prospective study of older men. J Electrocardiol. 2008;41(6):704-10.

24. Li X, Ren H, Xu ZR, Liu YJ, Yang XP, Liu JQ. Prevalence and risk factors of prolonged QTc interval among Chinese patients with type 2 diabetes. Experimental diabetes research. 2012; 2012:234084.

25. Solini A, Passaro A, D'Elia K, Calzoni F, Alberti L, Fellin R. The relationship of plasma glucose and electrocardiographic parameters in elderly women with different degrees of glucose tolerance. Aging. 2000;12(4):249-55.

26. Dimova R, Tankova T, Guergueltcheva V, Tournev I, Chakarova N, Grozeva G, Dakovska L. Risk factors for autonomic and somatic nerve dysfunction in different stages of glucose tolerance. J Diabetes Complicat. 
2017;31(3):537-43.

27. Orosz A, Baczko I, Nyiraty S, Korei AE, Putz Z, Takacs R, Nemes A, Varkonyi TT, Balogh L, Abraham G, et al. Increased Short-Term Beat-to-Beat QT Interval Variability in Patients with Impaired Glucose Tolerance. Front Endocrinol. 2017;8:129.

28. Brown DW, Giles WH, Greenlund KJ, Valdez R, Croft JB. Impaired fasting glucose, diabetes mellitus, and cardiovascular disease risk factors are associated with prolonged QTc duration. Results from the Third National Health and Nutrition Examination Survey. J Cardiovasc Risk. 2001;8(4):227-33.

29. van Noord C, Sturkenboom MC, Straus SM, Hofman A, Kors JA, Witteman JC, Stricker BH. Serum glucose and insulin are associated with QTc and RR intervals in nondiabetic elderly. European journal of endocrinology. 2010;162(2):241-8.

30. Tesfamariam B, Brown ML, Cohen RA. Elevated glucose impairs endothelium-dependent relaxation by activating protein kinase C. J Clin Investig. 1991;87(5):1643-8.

31. Davis FB, Davis PJ, Nat G, Blas SD, MacGillivray M, Gutman S, Feldman MJ. The effect of in vivo glucose administration on human erythrocyte Ca2+-ATPase activity and on enzyme responsiveness in vitro to thyroid hormone and calmodulin. Diabetes. 1985;34(7):639-46.

32. Santulli G, Pagano G, Sardu C, Xie W, Reiken S, D'Ascia SL, Cannone M, Marziliano N, Trimarco B, Guise TA, et al. Calcium release channel RyR2 regulates insulin release and glucose homeostasis. J Clin Investig. 2015;125(5):1968-78.

33. Fiorentini A, Perciaccante A, Valente R, Paris A, Serra P, Tubani L. The correlation among QTc interval, hyperglycaemia and the impaired autonomic activity. Autonomic neuroscience: basic clinical. 2010;154(12):94-8.

34. Alberti KG, Zimmet PZ. Definition, diagnosis and classification of diabetes mellitus and its complications. Part 1: diagnosis and classification of diabetes mellitus provisional report of a WHO consultation. Diabetic medicine: a journal of the British Diabetic Association. 1998;15(7):539-53.

35. Decode Study Group EDEG. Is the current definition for diabetes relevant to mortality risk from all causes and cardiovascular and noncardiovascular diseases? Diabetes Care. 2003;26(3):688-96.

\section{Figures}




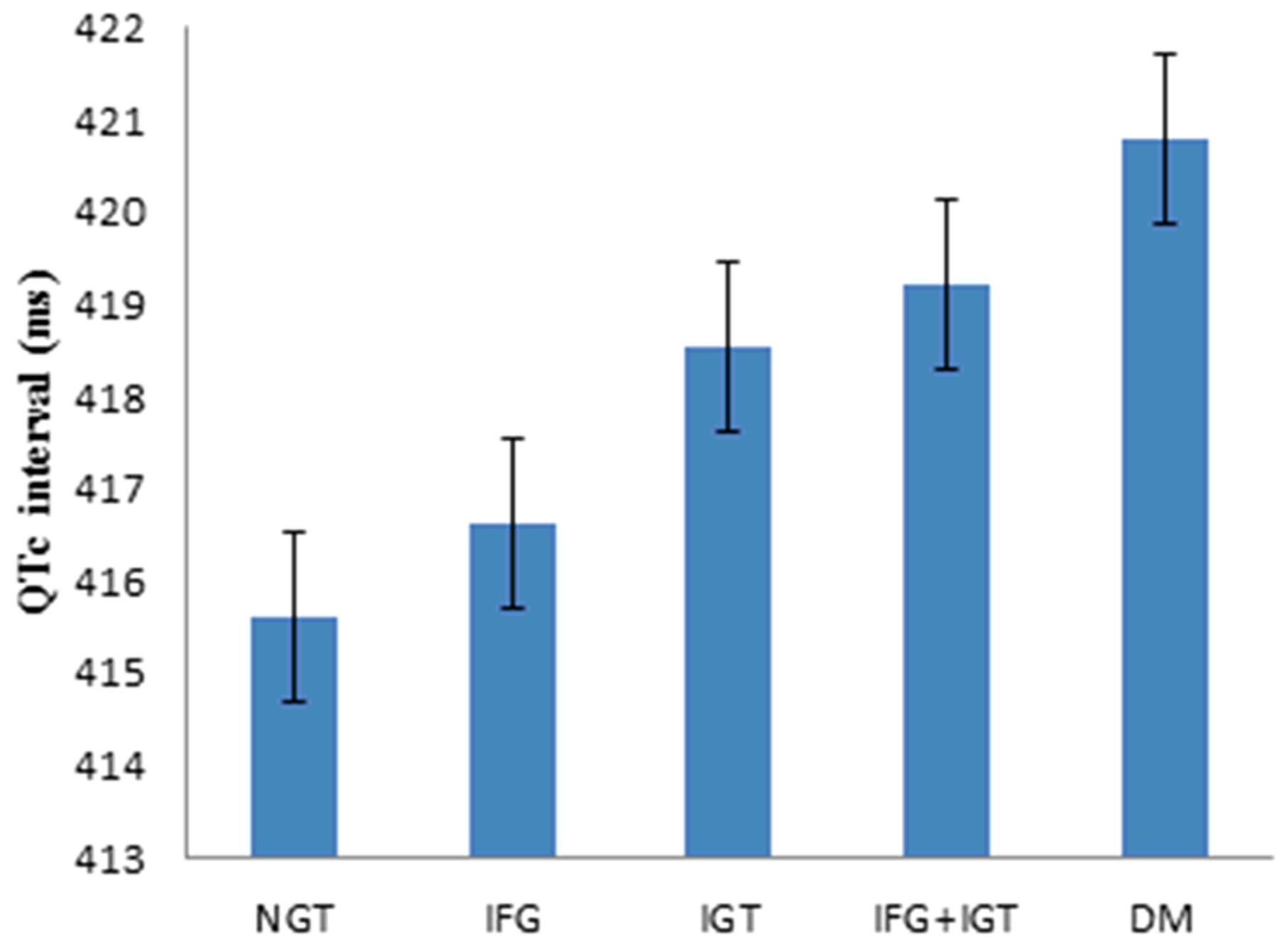

Figure 1

Adjusted means ( \pm SEM) of QTc interval in subjects with NGR, IGR (isolated IFG, isolated IGT), combined IFG/IGT and diabetes mellitus (DM). Subjects with type 2 diabetes mellitus and IGR (isolated IFG, isolated IGT and IFG/IGT) had higher level of QTc interval than those with NGR (both $p<0.0001)$. There was no significant difference among the subgroups of IGR $(p=0.35)$ and between the IGR and type 2 diabetes mellitus groups $(p=0.61)$ 